\title{
Model of neutral network sliding design by large envelope flight control law
}

\author{
Wei-Lun Chen $\dagger$ and Gong-Cai Xin \\ Department of Instrument and Electric Engineering \\ The First Aeronautical Institute of Air Force \\ Xinyang, Henan, China \\ †E-mail:sdweilun@163.com
}

\begin{abstract}
In this paper, the neural network sliding mode control design method is studied for the large enveloping flight control law of the model with very different flight parameters. The neural network theory is used to approximate the nonlinear system and eliminate the errors brought by the approximate inversion, and the residual error is solved by sliding model control. Therefore, the nonlinear model can be approximated accurately and the robustness and anti-jamming ability of the flight control system can be improved. The simulation results show that the design of the neural network - sliding model large envelope flight controller has excellent control performance.
\end{abstract}

Keywords: Adaptive Control; Nonlinear Control; Sliding Model Control.

\section{Introduction}

Pneumatic parameters change with altitude, mach numbers or dynamic pressure, and the moving range is very wide. The notable characteristic of new-generation fighter is high mobility, high agility, the wide moving range of velocity and altitude and continuous enlargement of the flight envelope. Here, the traditional design method of flight control can't satisfy the need of new-generation fighter to high performance mobile flight. It is the big difficulty to design flight control system. Non-linear dynamic inversion method based on neural network is introduced to design the large envelope flight control and get good effect. But the robustness and the transient characteristic performance of this control system is not strong to dynamic characteristic and external disturbance of non-model system $^{[1-4]}$.

A neural network-sliding model flight controller is designed to overcome the shortcoming above and increase the control precision in each area respectively, and continuous switch controllers by different flight areas. In the design of neural network-sliding model flight controller, on-line neural network realizes inversion of nonlinear system, and introduce sliding model control to 
improve adaptive control. Thereby it improves the robustness and transient characteristic performance. At last, the performance of the enlarge envelope flight controller is validated by the simulation of fighter envelope mobile flight.

\section{Question Description}

Airplane is a very complex system. And equation of motion of the newgeneration fighter is 6 degree-of-freedom and 12 states non-linear equation with each states variables existing interface coupling.

Each coefficients of nonlinear whole variable equation is determined by Pneumatic derivatives, and it changes with flight states $(H, M, \alpha$, aileron $\delta x$, rudder $\delta y$ and elevator $\delta z$ ). So in the design of flight controller, the uniform nonlinear model is used, if the Pneumatic derivatives can't cover the whole flight envelope, the control performance will be bad seriously. Therefore, flight envelope is divided into different areas by flight height and mach numbers, and the aircraft model need not be approximated in the whole envelope. So it can approximate aircraft model accurately. At the same time, to each area, design control system as shown in fig. 1 respectively. In the course of flight, switch the different control systems by different areas.

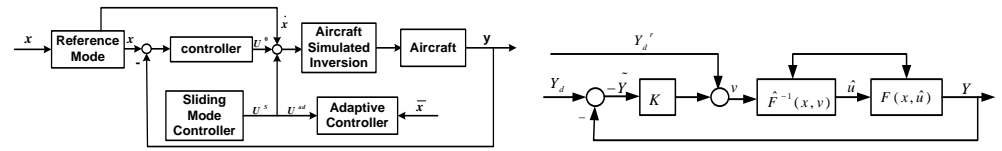

Fig.1. Block Diagram of Control SystemFig.2. Approximate Dynamic Inversion Control

\subsection{Design of large envelop neural Network-sliding model flight controller}

Division of Flight Envelop. It is a big difficulty to get the inversion of large flight envelop nonlinear model by neural network. So the flight envelop is divided into different areas. It becomes easy that Each area gets the inversion of nonlinear model by neural network respectively.

Inversion of Nonlinear Model Based Neural Network. Get the inversion of 6 degree-of-freedom full variable nonlinear model directly to new-generation fighter by neural network.Consider the following second-order nonlinear system:

$$
\begin{array}{r}
\ddot{x}=f(x, \dot{x}, \delta) \\
\psi=\eta(\xi)
\end{array}
$$

Where $\mathrm{x}$ is state variable; $\delta$ is control variable; $\mathrm{y}$ is output; $x, \dot{x} \in R^{n}, \delta \in R^{m}, m \geq n, y \in R^{p}$. The control task is to get the control variable $\delta$ 
which satisfies the requirements of the output $y$. Now, gets derivative for equation (2)

$$
y^{\prime}=\left[\begin{array}{c}
y_{1}{ }^{\left(r_{1}\right)} \\
y_{2}{ }^{\left(r_{2}\right)} \\
\vdots \\
y_{p}{ }^{\left(r_{p}\right)}
\end{array}\right]=F(x, \dot{x}, \delta)=v
$$

Then $\delta=F^{-1}(x, \dot{x}, v)$. For any output subsystem: ${ }^{y_{i}^{\left(r_{i}\right)}}=v_{i}(\mathrm{i}=1,2, \ldots, \mathrm{p})$. Here, viis the pseudo-control variable.

We can see, non-linear features are turned into the linearization. However, it is the calculation under ideal circumstances. But inverse calculation errors and external disturbances still exist. Therefore, equation (3) can be turned into $\ddot{x}=f(x, \dot{x}, \delta)+\Delta(x, \dot{x}, \delta), \Delta(x, \dot{x}, \delta)$ is the system random error. On the other hand, if the mathematical model is not accurately knowable, let the derivative of $F(x, \dot{x}, \delta)$ equal $F^{\prime}(x, \dot{x}, \delta)$, so $\delta$ can be changed into $\delta^{\prime}=F^{-1}(x, \dot{x}, v)$, we can see, the nonlinearity can not be fully offset. And its control block diagram is shown in Fig. 1. Where $F^{-1}(x, v) F(x, \hat{\delta})=I$.

The essence of the nonlinear dynamic inversion integration system is to counteract the nonlinearity of controlled objects by means of non-linear inversion and nonlinear function. When the object model is inaccurate, nonlinearity cannot be counteracted reasonably, and system robustness cannot be guaranteed. Therefore, a suitable model error compensation is required to deal with model uncertainty. On the one hand, it is necessary to compensate for external disturbances; On the other hand, it needs to deal with modeling errors. Adaptive control [2] is the primary means of dealing with model uncertainty, but it can only handle the uncertainty of object parameter.

\subsection{Neural network adaptive control}

In this paper, Lewis proposed multi-layer perceptron neural network to reconfigure the inversion error, and neural network structure is shown in reference [1], its input-output mapping relationship can be written as

$$
y_{i}=\sum_{j=1}^{N_{2}}\left[w_{i j} \cdot \sigma\left(\sum_{k=1}^{N_{1}} v_{j k} x_{k}+\theta_{v j}\right)+\theta_{w i}\right], \quad i=1,2, \cdots, N_{3}
$$

Where $y_{i}$ denotes the hidden layer activation function; $v_{j k}$ denotes the connection weights between the input layer and hidden layer; $\mathrm{w}_{\mathrm{ij}}$ denotes the connection weights between the hidden layer and output layer, $\theta_{\mathrm{vj}}, \theta_{\mathrm{wi}}$ are 
neuron threshold; $\mathrm{N}_{1}, \mathrm{~N}_{2}, \mathrm{~N}_{3}$ is the neuron number for the input layer, hidden layer, output layer respectively.

If the enough input information and the number of hidden layer neurons are given, neural network can approach continuous nonlinear functions with any accuracy. Therefore, for continuous uncertain nonlinear inversion error function $\Delta(x, \dot{x}, \delta)$ and the inversion system standard error $\varepsilon_{\mathrm{N}}>0$, there are $\mathrm{N}_{2}$ and network

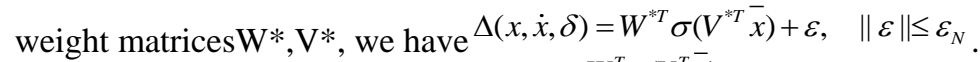

the adaptive items are taken as $v_{a d}=W^{T} \sigma\left(V^{T} \bar{x}\right)$, we can get the approximate performance of nonlinear function by adjusting their weights, and minimize errors.

\subsection{Neural network adaptive control and sliding mode control integrated}

From above, we can see, neural network adaptive control is a step-by-step learning process in the course of system running, this characterization determines that it can only deal with the steady or gradient uncertain model, and these uncertainties must also be expressed with a group of the unknown parameters, but it cannot deal well with rapidly changing parameters and the dynamics systems which have not constructed model. Therefore, this paper introduces sliding mode control to improve the fuzzy neural network adaptive control.

The design ideas are: take the sliding surface of subsystem $\mathrm{j}: s_{j}\left(\tilde{y}_{j}, t\right)=\tilde{y}=0$, let $\dot{s}_{j}=0$, we can get solution of equivalent pseudo-control $\hat{v}_{j}=\hat{\dot{y}}_{j}=0$. Define ${ }^{\rho_{j}^{*}}$ as residual error, and take $\bar{v}_{j}=\rho_{j} \operatorname{sgn}\left(s_{j}\right)$ as sliding robust compensation term. Let $\tilde{\rho}_{j}=\rho_{j}-\rho_{j}^{*}$, under the pseudo-control condition,

$$
\dot{s}_{j}=\Delta_{j}-v_{a d j}-v_{j}-k_{1} \dot{s}_{j}=0
$$

Define the Lyapunov function

$$
V_{j}=\frac{1}{2} s_{j}{ }^{2}+\frac{1}{2 \lambda_{j}} \tilde{\vartheta}_{j}^{T} \tilde{\vartheta}_{j}+\frac{1}{2 \eta_{j}} \tilde{\rho}_{j}^{2}
$$

Then

$$
\bar{V}_{j}=s_{j}\left[-\tilde{\vartheta}_{j}^{T} \rho(z)+\varepsilon_{j}-\rho_{j} \operatorname{sgn}\left(s_{j}\right)-k_{1} s_{j}\right]+\frac{1}{\lambda_{j}} \tilde{\vartheta}_{j}^{T} \tilde{\vartheta}_{j}+\frac{1}{\eta_{j}} \tilde{\rho}_{j} \dot{\tilde{\rho}}_{j}
$$

The adjustment rules of $\tilde{\vartheta}_{j}, \rho_{j}$ are shown $\dot{\tilde{\vartheta}}_{j}=\dot{\vartheta}_{j}=\lambda_{j} s_{j} \rho(z) \quad \dot{\tilde{\rho}}_{j}=\dot{\rho}_{j}=\eta_{j}\left|s_{j}\right|$

We can see, ${ }_{j} \leq 0$, so the system is wholly stable. To eliminate the buffeting, compensation term of robust sliding mode as follows: 


$$
\bar{v}_{j}=\rho_{j} \operatorname{sat}\left(\frac{s_{j}}{\Phi_{j}}\right)
$$

When the system begin to operate, the system trajectory leaves the boundary layer of the sliding surface, and adaptive neural network system based on sliding model compensation starts to work, then the system trajectory quickly back to the inner of boundary layer of the sliding surface, at this time, the adaptive control process is cut off. If the system trajectory leaves again, repeat this process until the sliding mode compensation term can fully compensate residual errors.

\subsection{Control system design}

Control system block diagram is shown in Fig.3.

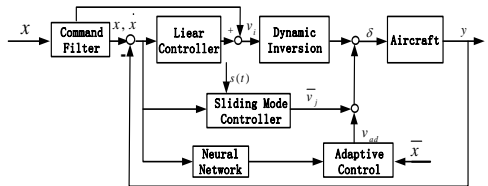

Fig.3. Control System Block Diagram

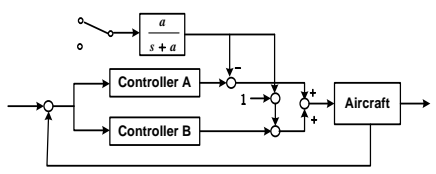

Fig.4. Control Law Switch

\subsection{Control law design}

(1) For any output $y_{j}(j=1,2, \ldots, p)$, derivative on $\delta$ until it explicitly appear in the expression;(2) If $\sum_{j=1}^{p} r_{j}=n$, the system can be linearized; If $\sum_{j=1}^{p} r_{j}<n$, study the stability of the inside dynamics subsystems;(3) To perform approximately linearization with dynamic inversion theory;(4) Defines the sliding surface: $s(\tilde{Y}, t)=\tilde{y}^{(r-1)}+k_{r} \tilde{y}^{(r-2)}+\cdots+k_{2} \tilde{y}=0 \quad ;(5)$ Determine the adaptive control structure, find adaptive items by neural networks;(6) Find sliding mode robust compensation item ${ }^{\bar{v}_{j}}$ by sliding mode control theory;(7) Use Lyapunov function to educe adaptive adjustment rules, and eliminate the impact of parameter drift.

\subsection{The realization of switching flight control law}

When fighters span different flight envelop areas, controllers need to switch. Not only inertial delay intenerate switch technology avoids jumping when controller switch, but also the structure is simple and easily realizes, as follow in fig.4. when control law A switches to B, we should smooth transient course at the whole output of control law. Use the unit $\mathrm{a} /(\mathrm{s}+\mathrm{a})$ to make the mode A diminish gradually and introduce mode B fade in gradually. It have a good transient control effect. Therefore, in the course of system switch, the control law is $A(t) e^{-a t}+B(t)\left[1-e^{-a t}\right]$. 


\section{Simulation}

Assume that a certain aircraft is 6 degree-of-freedom full variable nonlinear model, flight envelop $(\mathrm{H}=0 \sim 15000 \mathrm{~m}, \mathrm{M}=0.6 \sim 1.6)$, initial flight altitude is $8000 \mathrm{~m}$, mach number is 0.8. Fig.5 is track curve of pitch angle. The real line is the large envelop control method designed in this paper, the broken line is general gain control method. We can see from the figure, in despite of the fighter model has bigger change, neural network-sliding mode controller still can make the system keep stable and no overshoot. But the track curve shakes three times up and down and the maximum overshoot is $5 \%$ by general gain control method. Fig.6 is the track curve of rolling angle. The real line is the large envelop control method designed in this paper. And the broken line is general gain control method. We can see from the figure, the control performance is better by neural network-sliding mode control method than general gain control method.
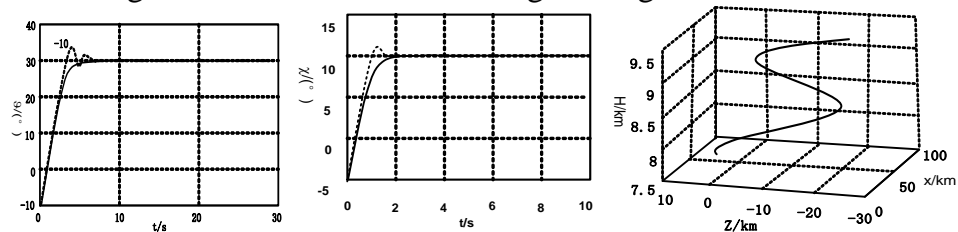

Fig.5. Track Curve of Pitch AngleFig.6. Track Curve of Rolling AngleFig.7. Screw Climb Track

Fig.7 is the screw climb track curve from the height $8000 \mathrm{~m}$. The parameters of fighter will occur big change with flight altitude and flight velocity. From the picture, we can see, the controller designed still has good control performance.

\section{Conclusion}

This paper introduced a kind of neural network-sliding mode large envelop control law design method. This method has stronger control performance and also it increases the adaptive robustness and transient characteristic performance. Compared with general gain control method, the simulation result that the aircraft designed by large envelop control still keeps stable and good control performance even if the flight parameters take great changes along with flight states in envelop.

\section{References}

1. Lewis F L, Yesildirek A, Liu K: Multilayer neural-net robot controller with guaranteed tracking performance [J]. IEEE Transactions on Neural Networks, 7(2):388-399(1996).

2. Kim B, Calise A J: Nonlinear flight control using neural networks [J]. Journal of Guidance, Control and Dynamics, 20(1):26-33(1977). 
3. Calise A J, Rysdyk R:Nonlinear adaptive flight control using neural networks[J]. IEEE Control System Magazine, 18(6): 14-25(1998).

4. McFarland M B, Calise A J: Adaptive nonlinear control of agile antiair missiles using neural networks[J]. IEEE Transactions on Control Systems Technology, 8(5): 749-756(2000).

5. Lin C M,MON Y J:Decoupling control by hierarchial fuzzy sliding mode controller[J].IEEE Transactions on Control Systems Technology, 13(4): 593-598 (2005). 